International Journal of Wireless \& Mobile Networks (IJWMN) Vol. 4, No. 2, April 2012

\title{
INTERFERENCE AVOIDANCE TECHNIQUES FOR Closed AcCess Small Cells Networks
}

\author{
Cristian Androne ${ }^{1}$ and Tudor Palade ${ }^{1}$ \\ ${ }^{1}$ Communications Department, Technical University of Cluj-Napoca \\ Cluj-Napoca, Romania \\ [cristian.androne, tudor.palade] dcom.utcluj.ro
}

\begin{abstract}
This paper investigates the impact of small cells networks deployment in a typical residential environment. First we will present some aspects with regard to small cells coverage area when deployed in a macrocell sector area. Using the observations with regards to coverage we will further investigate the deployment of a residential femtocell network, implemented in closed access form. The paper proposes two algorithms in order to reduce the interference level at the macrocell user site, considering that the operator owns at least two carrier frequencies which will be used for both the macrocellular and femtocellular layers. The performances of the two proposed algorithms are presented in comparison with the case when no interference mitigation methods are implemented. The obtained results show a significant improvement in the signal to interference and noise ratio, obtained at the user location.
\end{abstract}

\section{KEYWORDS}

Femtocell, Macrocell, Interference Mitigation, SINR, Small Cell, Residential Scenario.

\section{INTRODUCTION}

In the past few years, due to the saturation of the market caused by the offering of only voice services [1], cellular communication operators have directed their attention towards IP data based services [2]. Because of this, as shown in [1], the average revenues per user have increased for operators that offer enhanced data services. Even so, one important impediment towards this direction is represented by the fact that assuring coverage using the traditional macrocellular networks can be very costly, especially in areas with high density population, which have correspondingly higher demands in terms of service rate [3]. It is estimated that approximately $66 \%$ of all voice calls and $90 \%$ of all data transfers happen in indoor environments [4], that is why it is crucial to assure a good indoor coverage in this areas, not just for voice services, but also for data which become more and more important.

Recent surveys have shown that approximately $45 \%$ of home environments and $30 \%$ of business environments suffer because of weak indoor coverage [5]. That is why it is expected that a good indoor coverage and high quality of service will lead to increased revenues for operators and will enhance customer loyalty.

The traditional approach towards what concerns coverage assurance in the indoor environment is represented by the use of outdoor macrocellular base stations (MBSs). This techniques present a series of drawbacks which reveal the need for alternative solutions:

- It is inefficient to assure a good indoor coverage using the traditional approach. For example, in UMTS networks an indoor user will have to use more power in order to compensate the losses caused by the penetrations of the walls. This will lead to an increase in the overall cell noise which could limit the capacity of the network and/or the quality of the services provided.

DOI : 10.5121/ijwmn.2012.4219 
International Journal of Wireless \& Mobile Networks (IJWMN) Vol. 4, No. 2, April 2012

- A high capacity network will need a high number of base stations, which will lead to increased deployment costs for the operators. Moreover, with the increase of the number of base stations, the planning and optimization of the network will be harder to realize. A high density in base station transmitters will cause interference problems on the downlink, because of the superimposing of the coverage areas.

- In $3 \mathrm{G}$ and beyond wireless communication networks, the carrier frequency used will be $2 \mathrm{GHz}$ or higher, therefore the penetrations of the buildings will become an important problem. More than that, the performances of the networks will suffer important downfalls, since the data rates can not be guaranteed for indoor users because of the communication channel conditions. Therefore, the need to have superior modulation and coding schemes is absolutely necessary in HSDPA, WiMAX or LTE deployments .

A detailed description of the heterogeneous network architecture implemented according to the WCDMA standard is given in [6] and [7]. The authors of these papers describe each of the entities which compose the new small cell-macrocell architecture. The main novelty in this situation consists in introducing a new equipment, i.e. the Home NodeB Gateway (3GPP terminology), which has the role of managing the small cell base stations, more exactly: assuring a secure connection between the small cell base station and the core network (CN), providing support for paging and handover procedures, transparent transfer of Layer 3 messages between the User Equipment (UE) and core network A part of the interfaces which were developed in order to communicate with the new entity are defined in [8], [9] and [10]. For other mobile communications standards similar entities were implemented with the same basic role.

In order to compensate the limitations of outdoor macrocells, different approaches have been proposed, in order to enhance the signal level in the indoor environment. A first solution was to introduce antennas inside the buildings. Therefore, the Distributed Antenna System (DAS) was developed, which consists in deploying multiple antennas ,connected to a common base station, in such a way that the resulting coverage would be uniform [4]. Another solution proposed, especially to assure coverage inside tunnels and long corridors, was the deployment of the radiant cable [4]. A more recent approach refers to the implementation of small cells inside buildings in order to enhance both coverage and capacity of the network.

Depending upon the actual deployment scenario, the small cells can take many shapes, as presented by the authors of [11]: residential femtocell, enterprise femtocell and hot-spot or picocell. The residential femtocell, is characteristic for suburban areas, and the femtocell base station (FBS) acts as a cellular network access point which has the role of providing the radio resources to the users of the networks. It is similar in concept to the wireless access point used in local area networks, and therefore it is designed to be implemented by the user in a decentralized manner. It has a low transmit power of maximum $24 \mathrm{dBm}$ [12] and allows a number of up to 5 simultaneous calls and data sessions [4].

A second approach in small cells deployment options refers to the implementation of enterprise femtocells. This type of base stations is characteristic to the office or industrial environment. The femtocell base station has a maximum transmit power of up to $30 \mathrm{dBm}$ [12] and can support a maximum number of 16 simultaneous calls and data sessions [4]. If the residential femtocell has a stand-alone implementation, the enterprise femtocell is normally deployed in a network of femtocells, creating in this way a network that is similar to the macrocellular network.

The last deployment option considers outdoor small cells, often known as picocells. The maximum transmit power for this kind of base station is of $37 \mathrm{dBm}$ [12], and can support a variable number of users depending on the quality of service level which needs to be met. For 
International Journal of Wireless \& Mobile Networks (IJWMN) Vol. 4, No. 2, April 2012

the rest of the paper we will consider only the case of the residential femtocell, and the term femtocell and small cell will be interchangeably used.

Regardless of their deployment options, small cells offer, according to the authors of [13] and [14], certain advantages which put them in front of the traditional macrocellular networks:

- Because of the low transmit power, small cell base stations can be implemented with a much higher deployment density than macrocells, leading to a superior spectral efficiency.

- Because the small cells are located inside indoor environments, they provide an enhanced signal level, assuring a better and more uniform coverage of the scenario. Due to their low transmit power, and using the basic concept of spectrum reuse, small cells increase the overall network capacity. Also, because they are very close to the mobile terminals, this leads to a longer lifetime of the terminals batteries.

- Due to the fact that some of the indoor users are handled by the small cells, the macrocell base stations can redirect their resources in order to offer better services to the outdoor users.

- Reduced costs for the operator for the implementation and maintenance of the overall network. Because the main characteristics of the small cell concept is it's availability, the mobile user will experience higher quality of service (QoS) levels, at low prices.

One important technical characteristic when it comes to small cells deployments is represented by the way in which the carrier frequencies of the operators are shared among the macrocellular and femtocellular layer, respectively. In this sense, Figure 1 from [15] illustrates the three main options: the dedicated carrier approach, the shared carrier approach and the partially shared carrier approach. Next, the authors of [15], give detailed characterizations of the three implementation options. For our later experiments, we will consider the partially shared carrier approach. Related to the deployment options of small cells (residential femtocell, enterprise femtocell and pico-cell), as well as to the carrier implementation, the access mode to the femtocell defines the way in which the femtocellular layer acts upon the macrocellular layer. Therefore, the authors of [4] talk about two access modes to the small cell: open and respectively closed access mode. The open access mode is characterized by the fact that all users are allowed access to the small cells resources. In this case the femtocell network acts as an extension to the macrocellular layer, enhancing the coverage and the capacity of the overall network.

On the other hand, when considering the closed access mode, the femtocell forbids access to the resources to all users which are not listed in the CSG (Closed Subscriber Group) list [4]. This access list contains the IMSI codes of the users which rightfully belong to the femtocell. This access mode is particularly specific to the residential femtocell, but it is not limited to this deployment option. The small cell base stations operating in closed access mode represent an important interference source upon the other macrocellular users which are not allowed to access the femtocell. This is due to the fact that these users are not connected to the strongest transmitter, but to one that is further away, usually a macrocell. According to recent surveys [4], the residential femtocell owners would like to have their equipment deployed in closed access mode, which can cause significant problems to the macrocellular layer if there aren't any interference mitigation methods implemented. This particular issue will represent the main theme of the paper in the next chapters.

The rest of the paper is organized as follows: in section 2 we will present the main simulation parameters that will be used later in the paper; in section 3 some aspects regarding small cell coverage area and dependency upon different parameters are studied; section 4 describes the residential scenario that will be implemented for the 
International Journal of Wireless \& Mobile Networks (IJWMN) Vol. 4, No. 2, April 2012

experiments; in section 5 the proposed algorithms for cross-layer interference avoidance are described and their performances analysed within the scenario previously described; finally section 6 draws the main conclusions of the paper and proposes future work for the interference avoidance methods.

\section{Small Cells Coverage Aspects}

The deployment of small cells is a process that started in 2009 for the third generation mobile communications networks [16], and now-a-days it is in full expansion so that during 2012 femtocells integrated in the LTE networks will become an important factor in commercial mobile networks. One important feature of the small cells base stations is represented by the coverage area that is about to be obtained with these transmitters, which depends on the implementation environment, on radio channel conditions and the availability of the equipment.

In the following chapter we will investigate the impact that small cells networks have upon the network users, from the point of view of their coverage area. The coverage radius of the femtocell base station is an important parameter that affects the capacity of the network on the downlink communication, on one side: the larger the coverage area of the femtocell, the more mobile users it will include managing to free a part of the macrocells resources; and on the uplink communication channel, on the other side, where the interference caused by the macrocell users upon the femtocellular layer can be so significant that it will block the communication at the small cell base station.

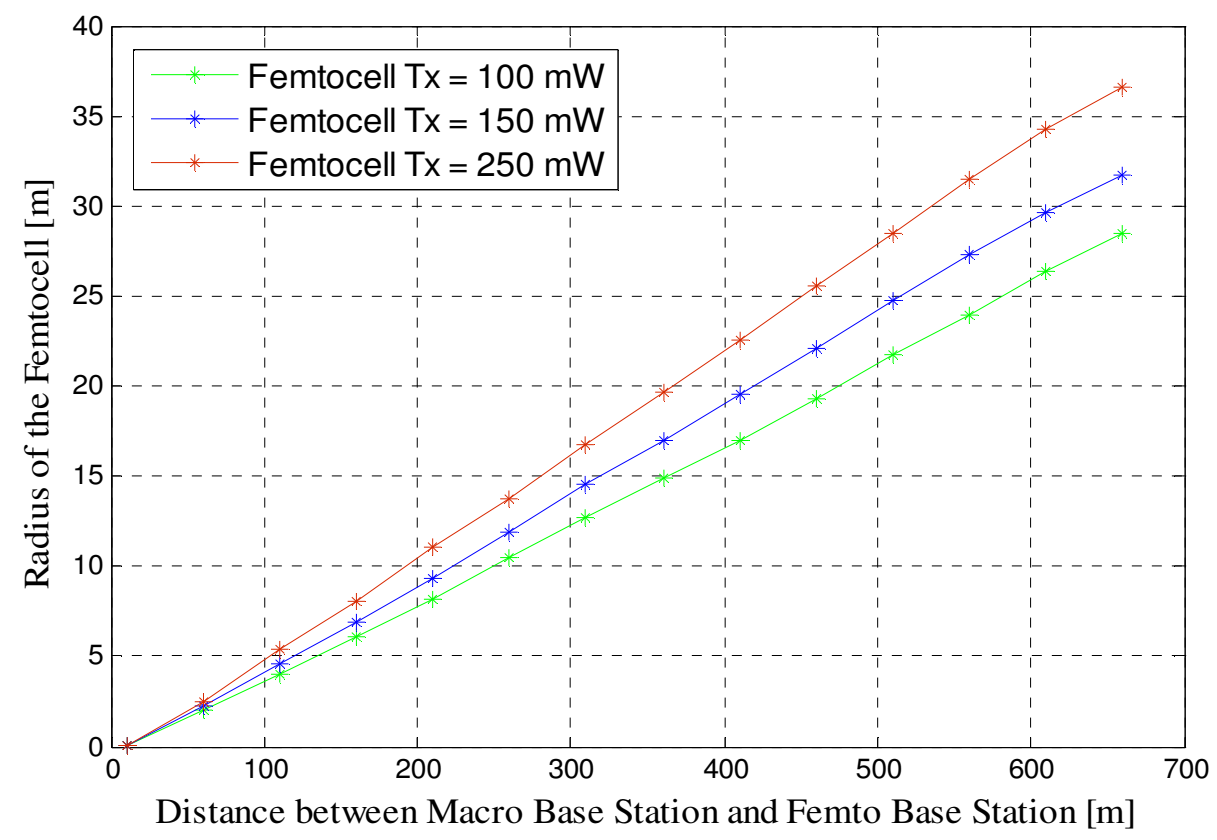

Figure 1. Coverage areas of residential femtocells vs The distance between MBS and FBS.

The larger the coverage area of the femtocell, the larger the minimum distance between the macrocell users and the FBS will be, which will lead to a decrease in the level of interference caused by the macrocellular user upon the femtocellular layer. In the following we will analyze the values of the coverage radii of the small cells base stations obtained for different transmit powers corresponding to the residential femtocells case. In this sense, Figure 1 offers a detailed view of the situation involved. 
International Journal of Wireless \& Mobile Networks (IJWMN) Vol. 4, No. 2, April 2012

Because small cells base stations are deployed within the coverage area of a macrocell, most often within a macrocellular sector, the coverage area of a femtocell depends on the level of signal coming from the macrocell base station. So, the higher the ratio between the signal coming from the femtocell and the signal coming from the macrocell, the more users the femtocell will manage to include. Figure 1 illustrates the variations of the coverage areas of the small cells in terms of the distance between the MBS and the FBS, for some typical values of the transmit powers of the residential femtocell.

So, as the FBS is moved away from the MBS, the signal from the latter one decreases, which will lead to an expansion of the small cell coverage area. The effect is more pronounced for a higher transmit power of the FBS.

For the calculation of the coverage areas of the small cells we considered an outdoor environment, without obstacles, for a better understanding of the impact that the distance between the two base stations has. For the link budget calculation only the path losses were taken into account, calculated according to relations given by Table 1 .

\begin{tabular}{|l|l|}
\hline $\begin{array}{l}\text { Path-loss } \\
\text { component }\end{array}$ & $\begin{array}{l}\text { Path-loss is modelled as } 11.81+38.63 \log _{10}(d) \text { for macrocell users and } \\
29.94+36.70 \log _{10}(d) \text { for small cell users where } d \text { is the distance from the } \\
\text { base station in meters. }\end{array}$ \\
\hline $\begin{array}{l}\text { Shadow } \\
\text { fading } \\
\text { component }\end{array}$ & $\begin{array}{l}\text { Shadow fading is modelled as spatially correlated random process with log- } \\
\text { normal distribution }(6 \mathrm{~dB} \text { standard deviation for the macrocell signal, } 4 \mathrm{~dB} \\
\text { standard deviation for the femtocell signal, spatial correlation } r(x)=e^{x / 20} \text { for } \\
\text { distance } x \text {. }\end{array}$ \\
\hline $\begin{array}{l}\text { Receiver } \\
\text { noise power } \\
\text { component }\end{array}$ & $\begin{array}{l}\text { The receiver noise power is modelled as } 10 \log _{10}(k T N F W) \text { where the } \\
\text { effective noise bandwidth is given as } W=3.84 \times 10^{6} \mathrm{~Hz} \text {, and } k T=1.3804 \times \\
10^{-23} \times 290 \mathrm{~W} / \mathrm{Hz} \text {. The noise figure at the } \mathrm{UE} \mathrm{is} N F_{[\mathrm{dB}]}=7 \mathrm{~dB} .\end{array}$ \\
\hline $\begin{array}{l}\text { Macrocell } \\
\text { antenna gain } \\
\text { component }\end{array}$ & $\begin{array}{l}\text { The macrocell antenna gain is calculated as } \\
G(\theta) d B=G_{\max }-\text { min }\left[12\left(\frac{\theta}{\beta}\right)^{2}, G_{s}\right], \quad-\pi \leq \theta \leq \pi \quad \text { with } \\
\beta=70 \pi / 180 \quad \text { angle where gain pattern is } 3 \mathrm{~dB} \text { down from peak } \\
G_{s}=20 \mathrm{~dB} \quad \text { sidelobe gain level in } \mathrm{dB} \\
G_{\max }=16 \mathrm{~dB} \quad \text { maximum gain level in } \mathrm{dB}\end{array}$ \\
\hline
\end{tabular}

Table 1. Simulation parameters.

The propagation model considered in the simulation is characterized by a log-distant path loss model which uses fixed and exponential coefficients defined according to [12]. The variation in amplitude caused by shadowing is often modelled as a log-normal distribution with a standard deviation defined according to the log-distant model. The fading maps have been generated according to the method described in [17] with the values of the standard deviation set according to the recommendation of [12].

Another important parameter that influences the coverage area of a femtocell is represented by the angular offset which is formed between the direction defined by the position of the FBS and the position of the MBS respectively, and the direction of the main lobe of the radiation pattern of the MBS antenna. Figure 2 illustrates the shapes and dimensions of the FBSs coverage areas 
deployed within a macrocellular sector. From Figure 2, one can observe the effect of the distance between the FBS and the MBS, as well as the effect of the angular offset.

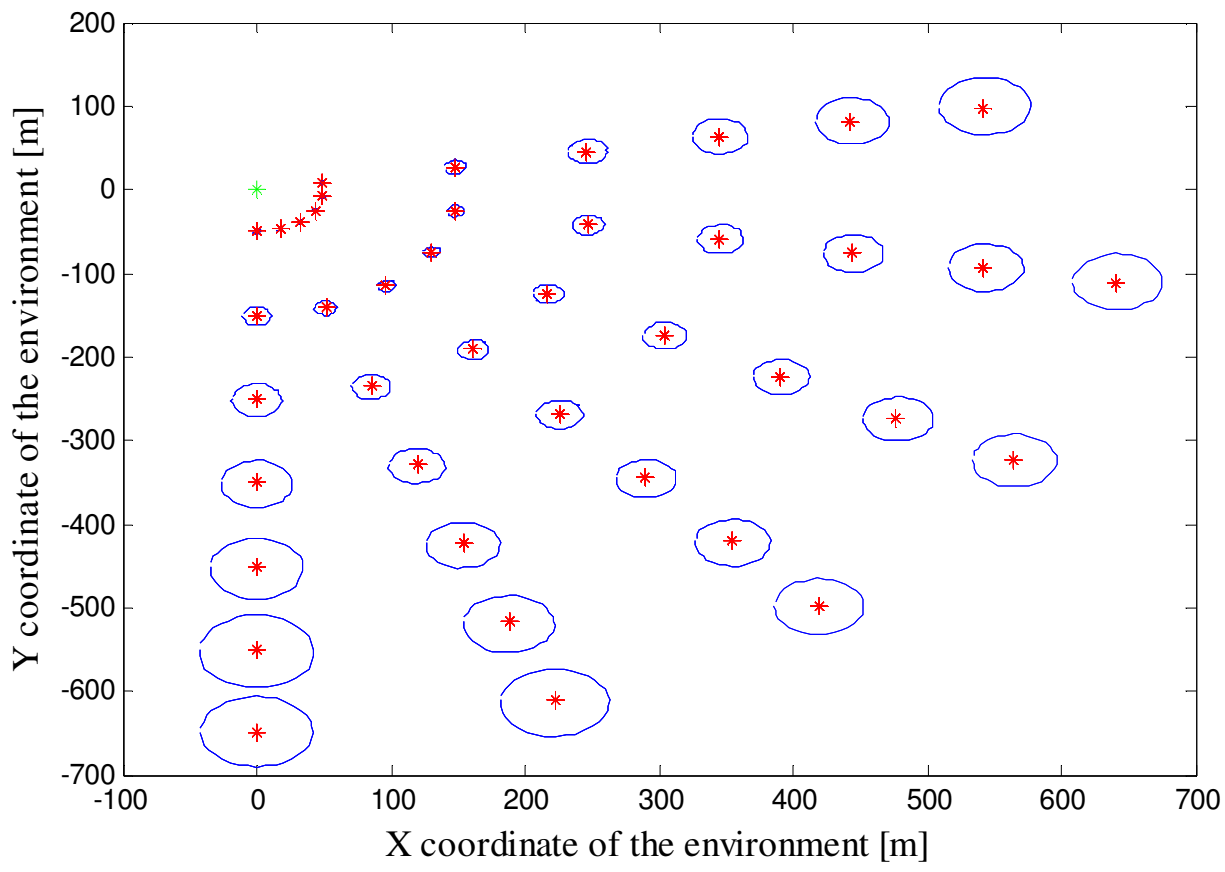

Figure 2. The deployment of small cells inside a macrocellular sector.

The small cell base stations deployed in the scenario have an ordered distribution with respect to MBS position, which is located at the $(0,0)$ coordinates, highlighted by the green star in the figure. The FBSs are deployed starting with a distance of 50 meters relative to the position of the MBS and with a distance of 100 meters between them, until they reach the edge of the macrocell which is at approximately 650 meters considering the same reference point. Their angular distribution within the macrocellular sector is made at 20 degrees angular offsets between the deployment directions.

One may observe an increase in the coverage area of the FBSs as the level of the signal coming from the MBS decreases, due to the distance between the MBS and respectively FBS positions, as well as due to the angular offset from the main lobe of the radiation pattern of the MBS antenna. The transmit power of the small cell base station is considered to be $250 \mathrm{~mW}$. For different values of the transmit power, the results are similar and vary according to the pattern defined by Figure 1 .

The red stars in the figure illustrate the actual positions of the small cell base stations. One may observe the fact that the shape of the coverage area is not symmetrical with respect to the position of the FBS, mainly because of the level of the signal coming from the MBS which is stronger towards MBS position and weaker in the opposite direction, which allows the coverage area of the femtocell to extend more in that direction.

The results illustrated by Figure 2 are obtained in an outdoor environment which doesn't take obstacles into account, in order to better show the effect of the angular offset. Also, in this case, for the link budget calculation we take into account only the path losses, defined according to Table 1. 
In Figure 3, on the other side, we illustrate the shapes and sizes of the femtocells in which we also take the shadow fading component into account, defined also by Table 1 . The deployment arrangement is the same as for the previous case, with distances of 100 meters between the FBSs and angular offsets of 20 degrees between the deployment directions. One may observe a more pronounced irregularity of the coverage areas, due to the shadow fading, defined as a lognormal distribution with a standard deviation of $6 \mathrm{~dB}$ for the macrocellular signal and respectively $4 \mathrm{~dB}$ for the femtocellular signal [12]. The pattern defined by Figure 2 is still valid here, in the sense that the coverage areas of the small cells increase with distance and with de shifting from the direction of the main lobe of the radiation pattern of the MBS antenna. The transmit power considered here for the small cell base station is of $250 \mathrm{~mW}$.

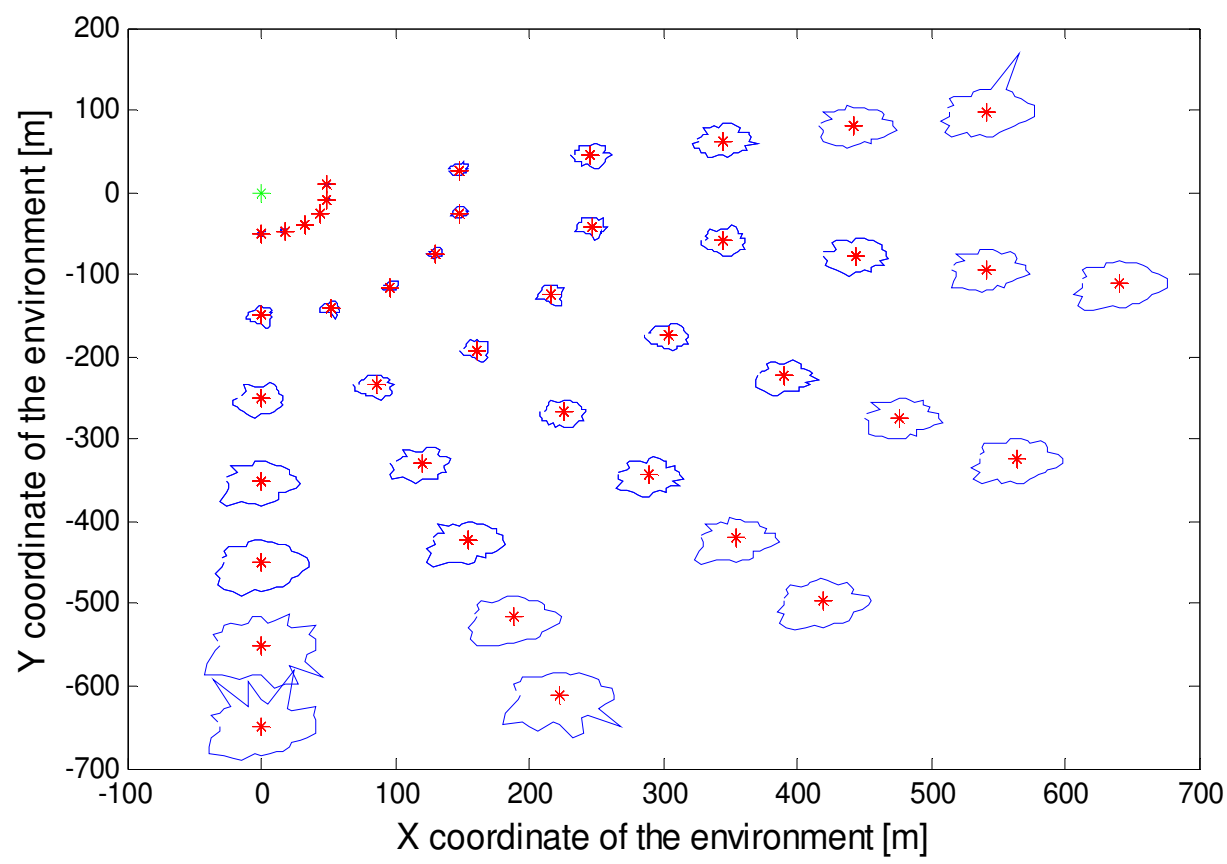

Figure 3. The deployment of small cells inside a macrocelullar sector with shadow fading.

The effect of shadow fading must be taken into account mainly when developing power control algorithms for the FBS, as well as for the mobile terminal, especially when it is handed over from the FBS to the MBS. This irregularity of the femtocells shape can determine weak performances of the power control algorithms if they are not flexible enough to overcome this effect. The higher the influence of the shadow fading upon the received signal, the more irregular the shape of the femtocell will be, which will lead to the necessity of power control and handover algorithms with higher performances. Therefore, Figure 4 illustrates the values of the difference between the maximum and respectively minimum level of the received signal strength from the FBS, on the downlink communication channel, in terms of the value of the shadow fading standard deviation, corresponding to the signal coming from the MBS. In order to fully understand the dependency upon the shadow fading standard deviation, we have illustrated graphs for different values of the shadow fading standard deviation of the signal coming from the FBS as well. 
International Journal of Wireless \& Mobile Networks (IJWMN) Vol. 4, No. 2, April 2012

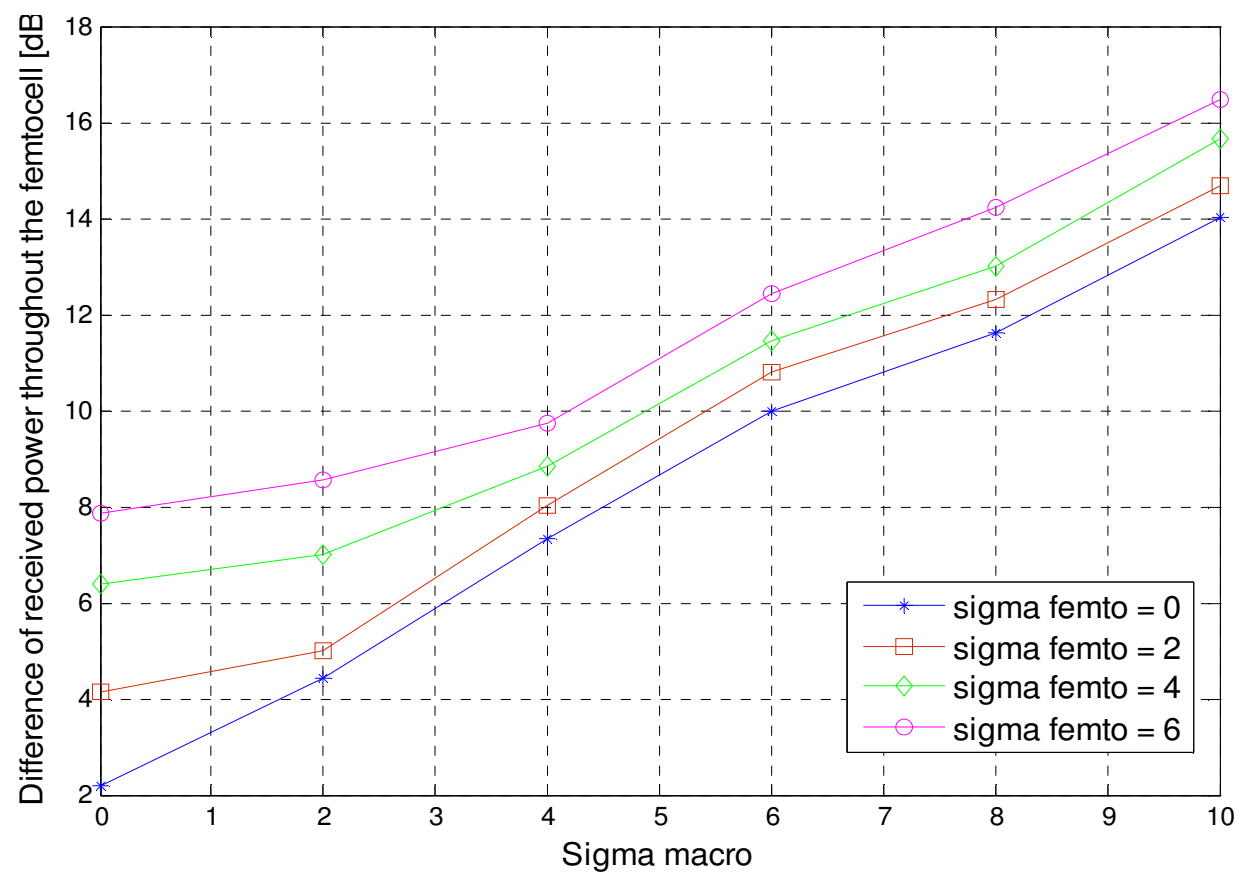

Figure 4. The difference in received signal strength coming from the FBS.

The graphs are obtained for a FBS transmit power of $250 \mathrm{~mW}$ and a positioning of it at half the maximum possible distance, i.e. half the distance between the MBS position and the macrocell coverage area edge. According to Figure 4 one may notice that there is a variation of the received signal strength coming from the femtocell even for the case when there is no shadow fading taken into account, an aspect which was validated by Figure 2 as well. The values presented in Figure 4 represent the average values obtained for that certain distance between the MBS and FBS, while varying the angular offset with respect to the direction of the main lobe of radiation of the MBS antenna, so that it sweeps the whole macrocellular sector.

As the levels of the shadow fading standard deviation increase for the signals coming from both macrocell and femtocell, so does the level of irregularity of the femtocell coverage area. For the extreme case when we consider the shadow fading standard deviation for the signal coming from the MBS to be $10 \mathrm{~dB}$, and the one coming from the FBS to be $6 \mathrm{~dB}$, the resulting value of the level difference in received signal strength is $16 \mathrm{~dB}$, with no differences in signal attenuations for different directions.

In Figure 5 we illustrate the difference between the maximum and respectively minimum level of the received signal strength coming from the FBS in terms of the distance between the MBS and FBS. The presented graphs are obtained for the case when we do not consider any shadow fading in the environment, and for the cases when the shadow fading standard deviation of the signal coming from the FBS is $2 \mathrm{~dB}$, while the one from the MBS is $6 \mathrm{~dB}$; the one from the FBS is $4 \mathrm{~dB}$ and the one from the MBS is $8 \mathrm{~dB}$ and respectively the one from the FBS is $6 \mathrm{~dB}$ and the one from the MBS is $10 \mathrm{~dB}$. The average value of each graph validates the aspects presented in Figure 4, proving that the higher the values of the shadow fading standard deviation, the higher the difference between the maximum and minimum levels of the received signal strength.

The evolutions of the graphs are distinct due to the various factors that influence their values. So, for the case when the shadow fading is neglected, at short distances between the MBS and FBS, the level difference is lower, due to the more symmetric shape of the femtocell with respect to the FBS position, following that it will increase with a value of maximum $2 \mathrm{~dB}$, a 
value that is practically insignificant from the point of view of the power control and handover algorithms. Therefore, for an environment without shadow fading, we can argue that the difference in received signal strength throughout the femtocell coverage area is approximately constant, with respect to the distance between the MBS and FBS.

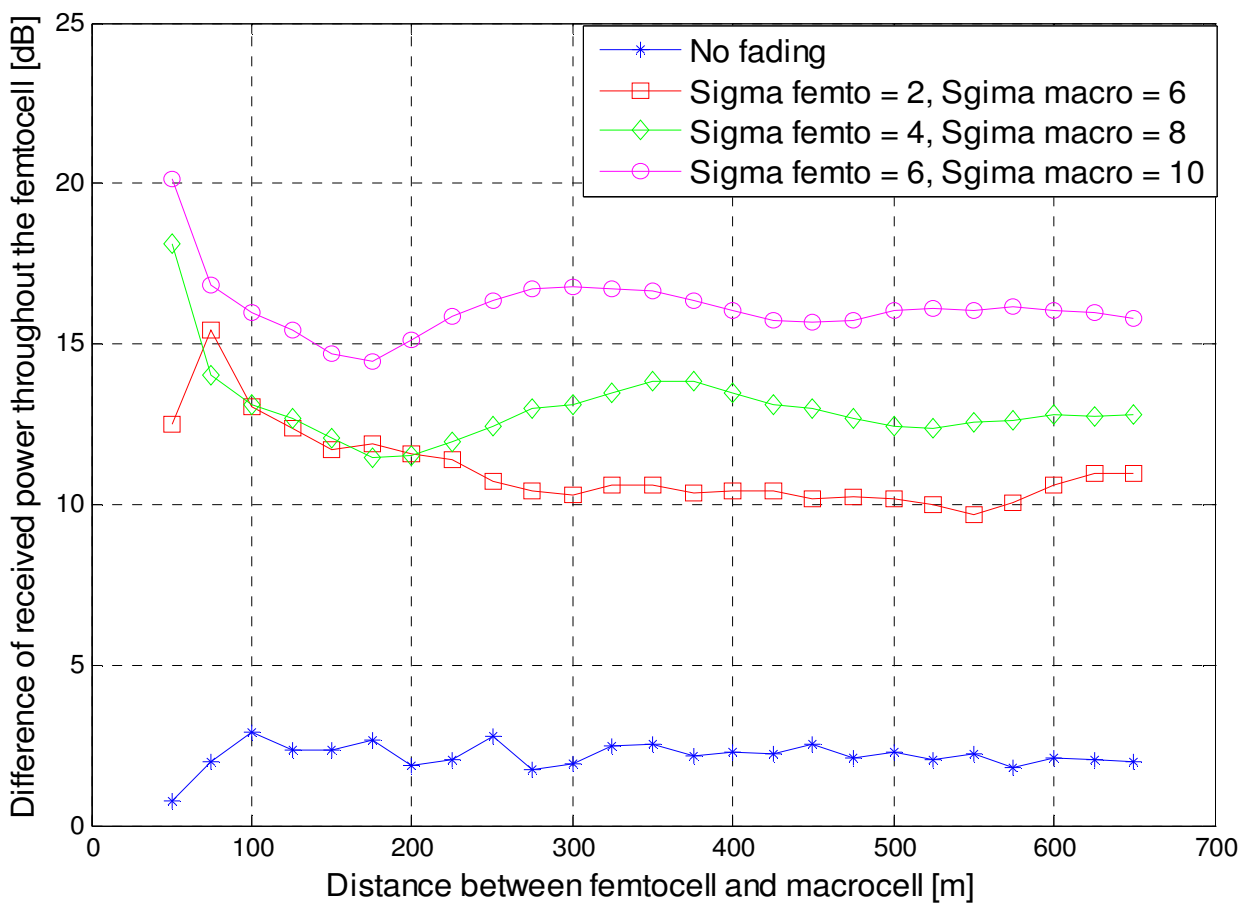

Figure 5. The difference in received signal strength as a function of the distance between the MBS and FBS.

If we consider environments in which shadow fading is present, according to Figure 5 we can argue that the difference in received signal level is higher for shorter distances between the MBS and FBS, due to the pronounced irregularity of the femtocell coverage area. This, corroborated with the fact that the signal level from any transmitter decreases with the square distance, makes the difference between the maximum and minimum received signal strength levels to be high in close vicinity to the MBS.

As the FBS get further away from the MBS, thus resulting in a larger coverage area, the effect of the irregularities decreases, this due also to the fact that the level of the received signal strength has a better constancy at longer distances from the transmitter. It can be observed that after approximately $30 \%$ of the maximum distance between the MBS and FBS, the variations tend to remain constant around a specific value.

\section{SimULATION SCENARIO}

In order to implement the interference mitigation algorithms of which we have spoken earlier in the paper, we need to define the environment scenario in which we will place out the two-layer mobile communications network. As mentioned before, we will consider the deployment of a residential femtocell network in a typical sub-urban environment. For this study we will use the same environment we have used in our previous work, [15] but we will consider a different approach from the user deployment point of view. The general view of the residential environment is presented in Figure 6. 
The simulation scenario consists of a street-like environment with houses placed on both sides of a road. The architecture of the houses is typical for the residential areas of the cities in the Transylvania area, Romania. Houses are symmetrically placed on both sides of the street which has a width of 20 meters. For larger scale scenarios we consider an additional backyard and garden for each house.

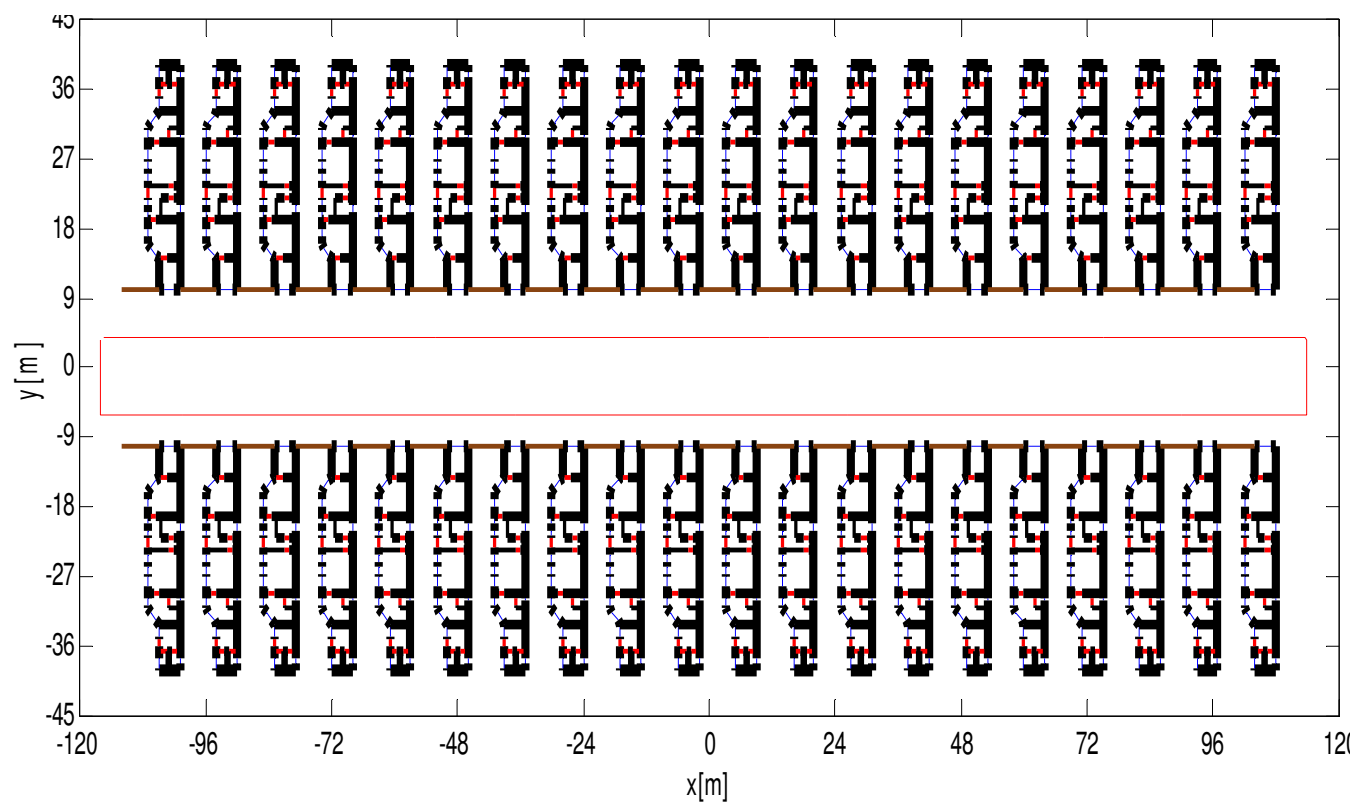

Figure 6. Simulation scenario.

The houses have one floor, with rooms mainly aligned one after the other in a train-like deployment. There are several entrances all of them being positioned on one side of the house. The footprint of the house is about 30 meters in length and 6 meters in width. For the construction of the houses several materials are considered, like concrete, brick, sheet-rock, glass and wood. The houses are equipped with entrance gates which are made of reinforced wood. The attenuations introduced by the construction materials, when considering perpendicular incidence angles of the waves, are presented in Table 2.

In the environment illustrated in Figure 6 we deploy a variable number of FBSs, with the condition that they are placed inside the houses and no more than one FBS is implemented in one house. The houses chosen are random, as well as their position inside the house. In our experiments we realized a sufficient number of simulation runs so that the position dependency of the FBSs influences as little as possible the final results.

The macrocellular layer is composed of 19 MBSs each of them equipped with three sectorial antennas. The MBS inter side distance (ISD) is equal to 1732 meters [12], as for a typical residential environment. The scenario previously presented in Figure 6 is placed at a distance of 750 meters from the central MBS, in a similar manner to the one presented in [15]. 
International Journal of Wireless \& Mobile Networks (IJWMN) Vol. 4, No. 2, April 2012

\begin{tabular}{|c|c|}
\hline House Element (Material used) & Attenuation introduced [dB] \\
\hline External wall (Concrete) & 15 \\
\hline Internal wall (Brick) & 10 \\
\hline Internal wall (Sheet-rock) & 7 \\
\hline House gate (Reinforced wood) & 5 \\
\hline Doors (Wood) & 3 \\
\hline Window (Glass) & 1 \\
\hline
\end{tabular}

Table 2. Attenuations introduced by the house elements.

The red line in Figure 6 represents the path followed by users that travel through the scenario. As we will later explain in the paper, we will consider several different paths for the users, and their closeness towards the houses varies from 1 meter to a maximum of 10 meters in some experiments.

\section{Algorithms DeSCRIPTION}

As mentioned earlier in the paper, most residential femtocell owners will most likely choose to have their equipment working in closed access mode. In this situation, the FBS will become a powerful interference source that may significantly degrade the performances of the macrocellular layer [15].

Considering the scenario previously described in the paper, a user that is passing through the environment may be seriously affected by the FBSs deployed in the nearby houses. A moving user may receive strong downlink signals from the near-by femtocells, but it can not connect to them, even if the femtocell signal is more powerful than the macrocell signal, because it is not allowed to do that. Therefore, under certain conditions it may even be rejected from the network because of its low channel quality indicators.

In order to avoid such a catastrophic scenario, we have proposed two carrier jumping algorithms which tend to solve the high interference problem by a mechanism which is often known as interference avoidance [4]. The two algorithms can be applied only for operators which have at least two carriers available for usage. The idea which stands behind the two proposals consists in a mechanism that triggers the macrocell to change its carrier once the downlink conditions for its travelling user degrade below a certain threshold.

The mobile terminal periodically reports its channel conditions to the host base station. The decisions regarding the change of carrier are based on these information averaged at every 10 ms. Considering the partially shared carrier deployment option described in [10], which considers that there are at least two carriers available, one which is used by both femtocellular and macrocellular layers and respectively one that is used only by the macrocells, the first algorithm that we propose is characterised by carrier jumping once the channel conditions fall below the considered threshold. Figure 7 illustrates the basic flow diagram of the algorithm.

The algorithm takes decisions at every $10 \mathrm{~ms}$ based on the previously three consecutive samples taken. If all of the three bins are below a certain threshold (SINR_thr), the algorithm triggers the MBS to change its carrier for that particular user. The carrier setting is kept for as long as the user is in that area or it is handed over to some other base station. Only if the logical AND of 
the three decisions is 1 , the algorithm will give the carrier jumping command, otherwise the MBS will keep its current carrier.

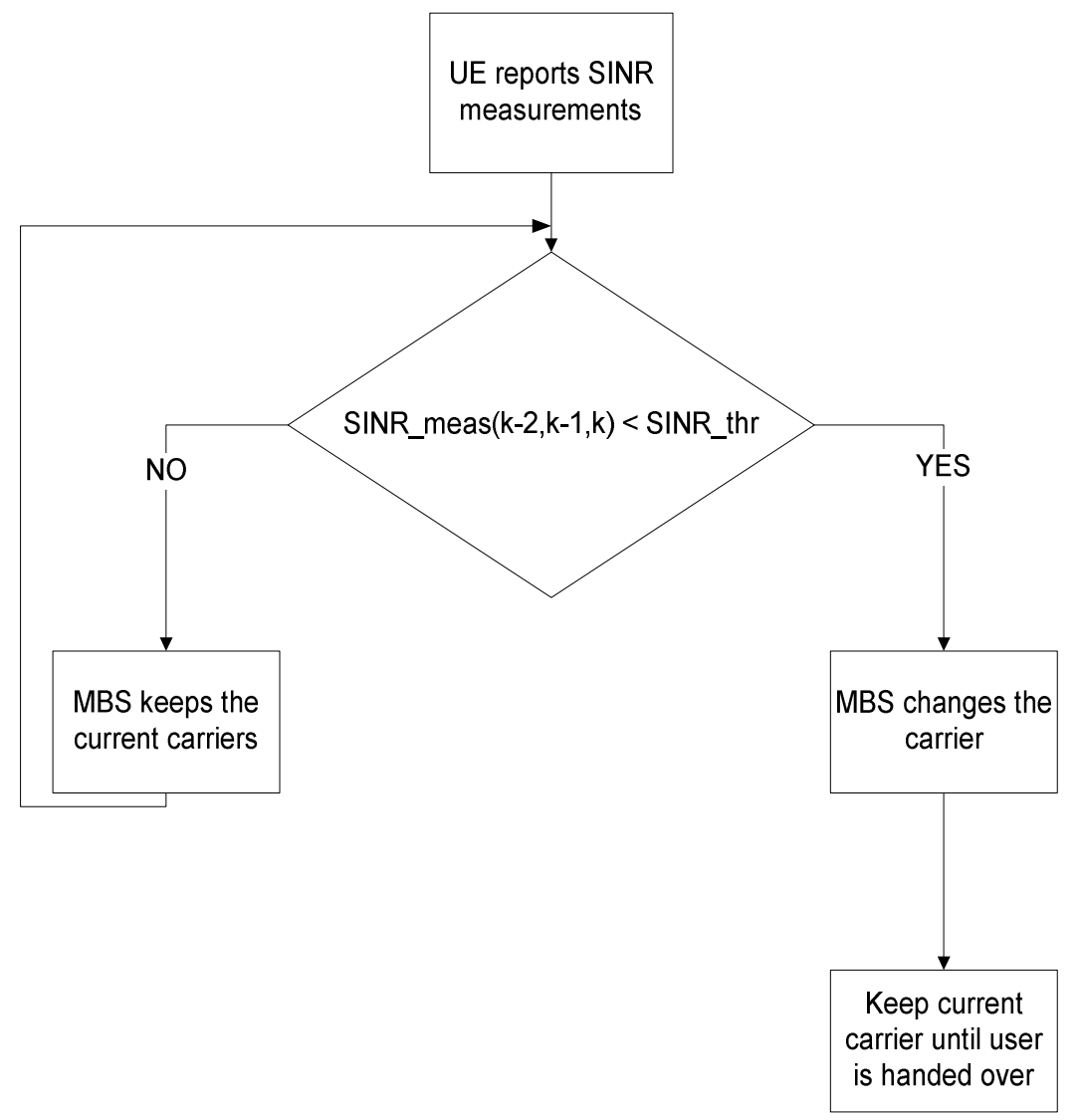

Figure 7. Carrier jumping algorithm1.

The algorithm presents the advantage of assuring a high SINR value for the travelling user. Once the channel conditions fall below the threshold, practically the user will experience a low SINR for $30 \mathrm{~ms}$, and then it will be transferred to a low interference environment, taken that the macrocellular layer was properly planned so that suitable performance indicators are met. However, in high user density environments, if we apply this algorithm and we will transfer all users on another carrier, this will tend to become a dedicated carrier approach with the femtocellular users one carrier and the macrocellular users on another carrier, which from the point of view of spectrum usage is highly inefficient.

Therefore, the second algorithm we propose is based on the same principle, but with the difference that once the macrocell changed its carrier for a particular user, after a predefined period of time it moves back to the original carrier and performs once again the channel conditions check. The carrier is then kept for as long as the channel conditions are not met for three consecutive samples, after that the user being moved again on the second carrier. The full flow diagram of the new algorithm is presented in Figure 8.

The advantage of this algorithm is that the user is not kept permanently on the new carrier, but the macrocell tries to move it back to the original carrier, thus resulting in a higher spectral efficiency. In this case, the second carrier can be seen as a safety band on which users with insufficient channel conditions can be brought in order to avoid a dropped call or data session. Thus most of the macrocells resources can be concentrated on the first carrier, while for the 
second one the resources can be assigned in such a way that it will support a limited number of users.

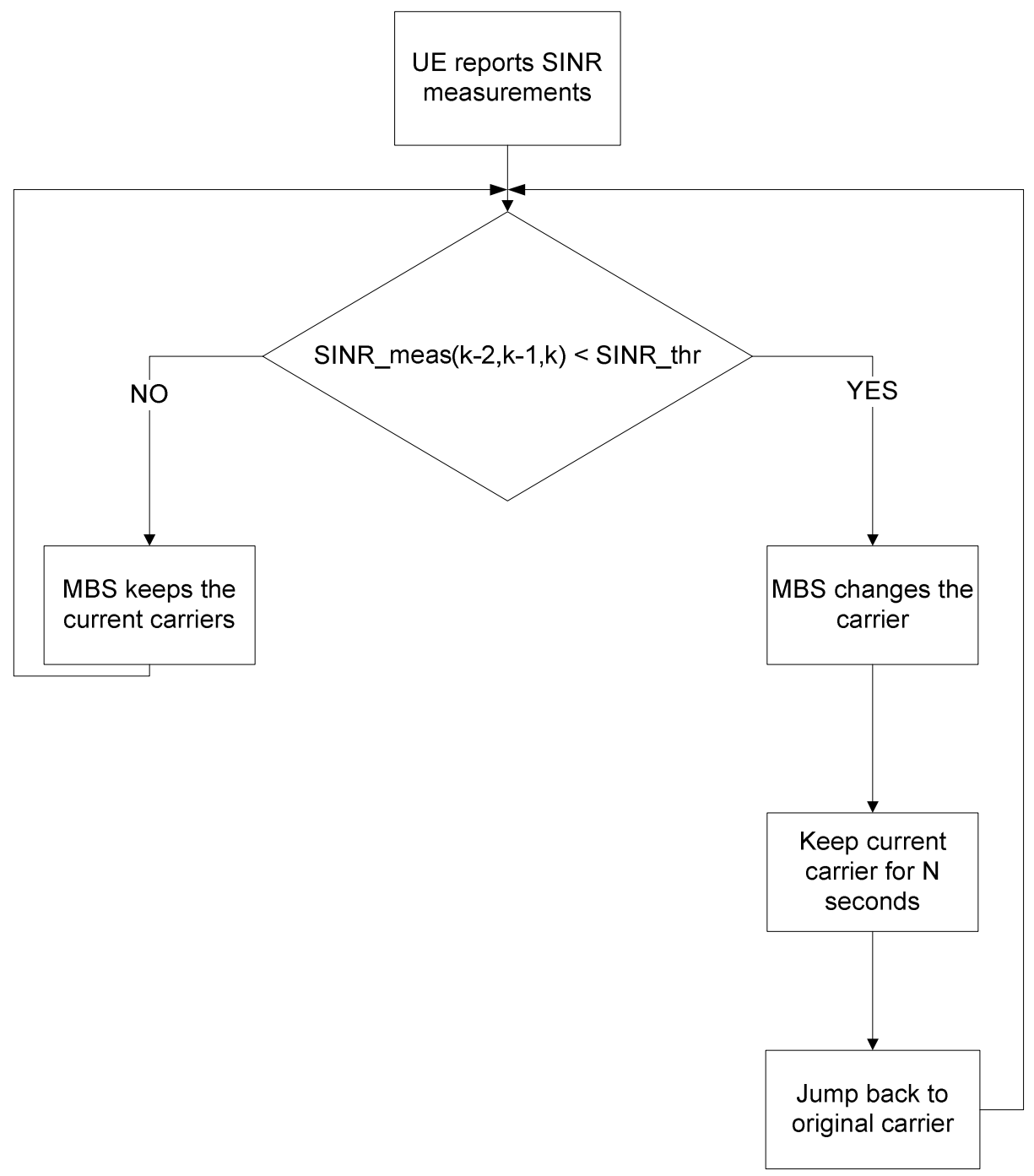

Figure 8. Carrier jumping algorithm 2.

In the following section of the paper we will investigate the performances obtained with the two algorithms compared to each other and with the case when no algorithm is implemented and the moving user is subject to high interference from the femtocellular layer.

\section{Simulation RESUltS}

Considering the two algorithms defined in the previous chapter, in this part of the paper, we will evaluate their performances in terms of parameters like the distance of the travelling user to the houses, the FBS deployment density, the transmit power of the FBSs, the speed of the travelling user and respectively the come-back time for the second algorithm. In order to see the impact of the variance of these parameters, first we will present in Figure 9 the general performances of the four deployment options: a purely macrocelullar network, a network with femtocells in which no interference avoidance techniques have been implemented, a network with femtocells 
for which Algorithm 1 was implemented and respectively, a network with femtocells for which Algorithm 2 was implemented.

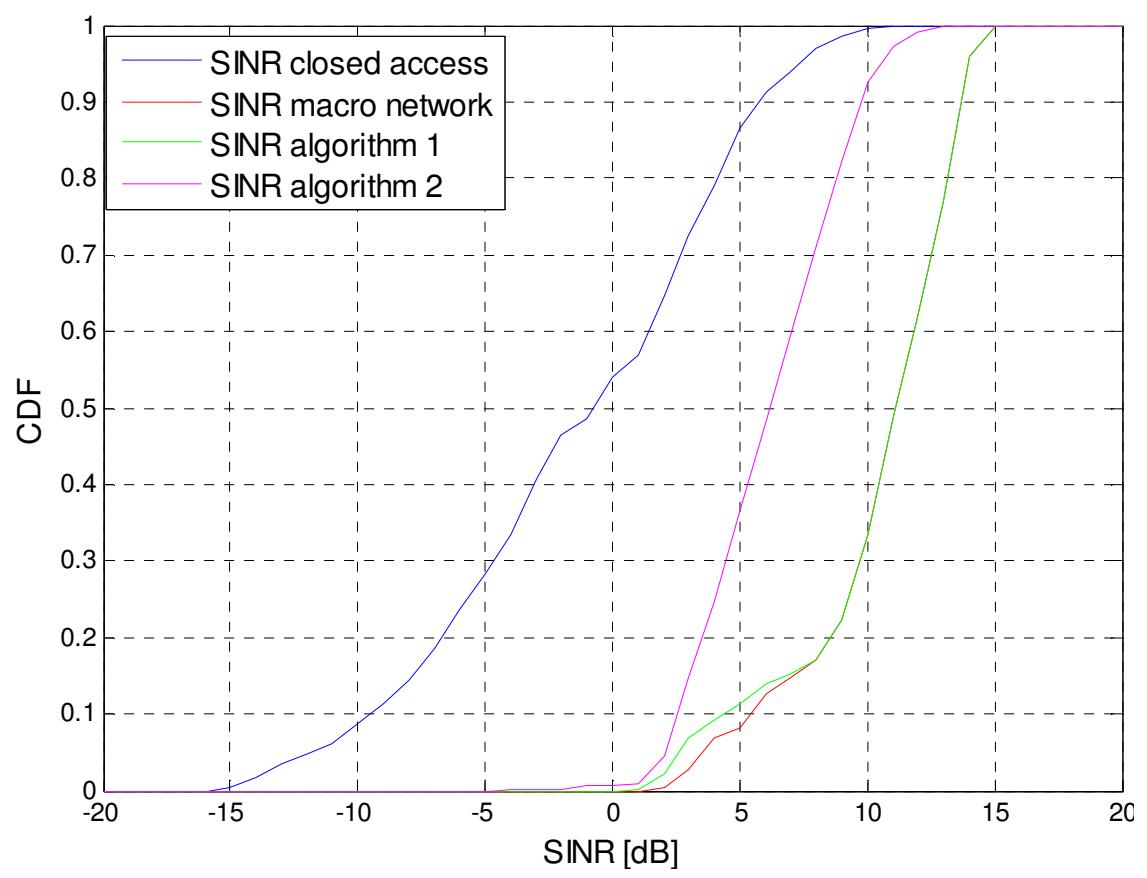

Figure 9. General performances of the deployment options.

One may see that the worst performances are obtained considering the closed access femtocell network with no interference avoidance techniques implemented. As expected, a purely macrocellular network will obtain the best performances in terms of reaching the lowest interference. Algorithm 1 obtains good performances, very close to the purely macrocellular network, because as soon as the network conditions imply a carrier modification, this is realized and the user is kept on that new carrier, limiting the interference.

Algorithm 2 doesn't realize such good performances, but the values obtained assure a proper QoS level for all the users, plus there is an enhanced spectrum efficiency. For this general view we considered a distance of 5 meters from the users travelling path to the houses, the transmit power for the FBSs is considered to be $250 \mathrm{~mW}$ and the travelling speed of the users is $1 \mathrm{~m} / \mathrm{s}$. In the case of Algorithm 2 the come-back time is considered to be 5 seconds, which will be the default values for these parameters in the following simulation scenarios. For the results in Figure 9 the fentocell density considered is of $220 \mathrm{FBSs} / \mathrm{km}^{2}$, but for the next experiments we will consider a default FBS density of $440 \mathrm{FBSs} / \mathrm{km}^{2}$.

Given that we have the average performances of the different deployment options, in the following part of the paper, we will observe the effect of each of the parameters previously enumerated upon the obtained SINR level for the cases when we have no interference avoidance methods, when Algorithm 1 is implemented and, respectively when Algorithm 2 is implemented. Therefore, Figure 10 illustrates the effect of the distance to houses on the SINR level obtained at the locations of a user which moves across the environment along a path similar to the one defined in Figure 6

. We have investigated the obtained SINR level for paths of shapes similar to the one in Figure 6 , defined along the environment houses, but for distances to the houses of $1 \mathrm{~m}, 5 \mathrm{~m}$ and 
respectively $10 \mathrm{~m}$. In this case we consider the default values for all the other parameters previously enumerated. Respecting the general performance chart, the worst case scenario is when there are no interference avoidance methods implemented.

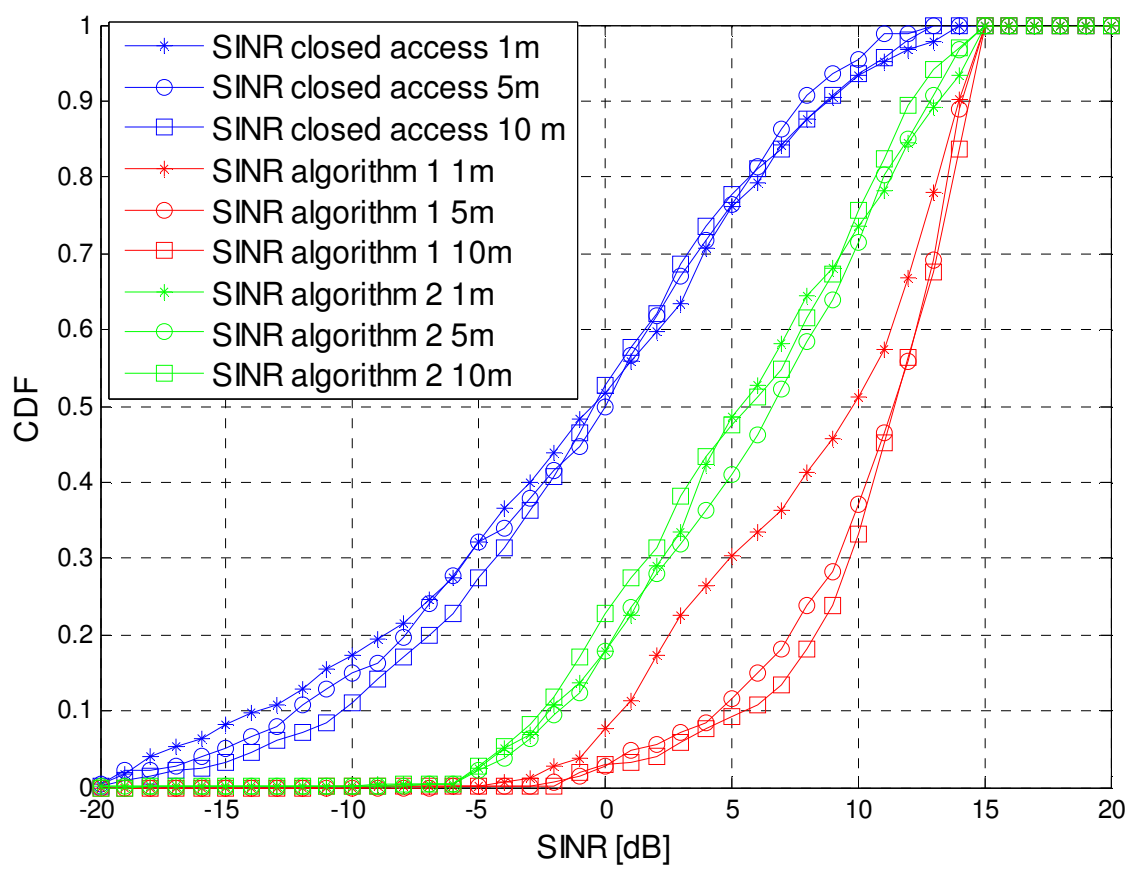

Figure 10. Effect of the distance to houses.

For this case, the closer the path is to the houses, the more powerful the negative impact is. This closeness is visible for the case of the Algorithm 1 implementation, when the distance to the houses affects the performances of the network. As soon as the path is shifted away from the houses, the travelling users obtain higher SINR values.

With the implementation of Algorithm 2, the position dependency of the obtained SINR level is significantly decreased, in this case there is no visible differentiation between the performances obtained for the three distances to the houses, which makes Algorithm 2 a viable solution irrespective of the position of the travelling user. Considering a minimum required SINR level of $-6.5 \mathrm{~dB}$ [10], in order to assure the desired QoS level for the users, both proposed algorithms meet these requirements and can be successfully implemented in real life scenarios.

Next, we will observe the effect of the FBS deployment density upon the level of the obtained SINR, at the positions of the travelling users. In this sense, Figure 11 illustrates the obtained performances for four different FBSs deployment densities. The lowest density corresponds to the deployment of a FBS in every eight houses, while the highest density corresponds to the deployment of a FBS in every two houses. The rest of the parameters have the default values previously mentioned.

One may notice the high impact of the FBS deployment density upon the obtained level of the SINR, when there are no interference avoidance methods implemented. Basically there is a difference of about $10 \mathrm{~dB}$ in the SINR level between the lowest and respectively highest deployment densities. Even with the lowest density, the performances obtained are still below the threshold of $-6.5 \mathrm{~dB}$. An important fact that needs to be added here is that we consider that a 
network performs within the required target if at least $99 \%$ of the SINR values obtained at the user position are above the threshold.

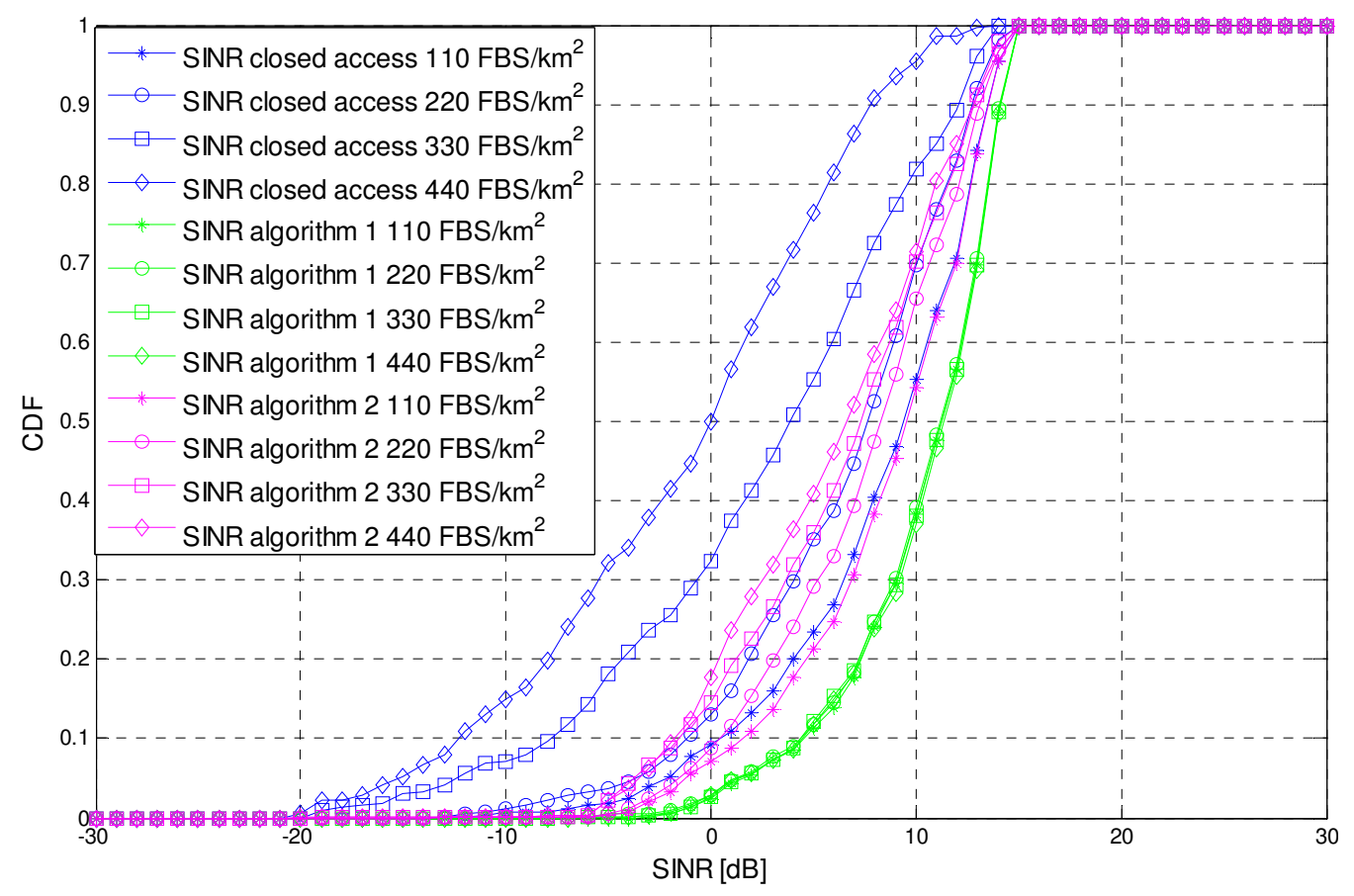

Figure 11. Effect of the FBS deployment density.

The femtocell density has little impact when we consider the implementation of Algorithm 1, simply because it commands the carrier jump, once the channel conditions fulfil the requirements and keeps the user on that carrier for the rest of the session. For the implementation of Algorithm 2, there is an effect of the FBS density, but the differences go down to about $5 \mathrm{~dB}$. Even so, both algorithms meet the QoS requirements.

Another important parameter that influences the level of the obtained SINR level at the user position is the FBS transmit power. In our experiment we have considered the same transmit powers for all the FBS, throughout one simulation case. Therefore, we take into account three transmit power: $100 \mathrm{~mW}, 150 \mathrm{~mW}$ and respectively, $250 \mathrm{~mW}$. These values are within the maximum allowed transmit powers for the residential femtocell, and offer a wide view of the phenomenon involved. Figure 12 presents the effect of the FBS transmit power upon the level of the obtained SINR.

Taking into consideration the trend of the previous results, the worst case scenario is for considering the closed access mode with no interference avoidance methods implemented. Within this set of results, using a FBS transmit power of $250 \mathrm{~mW}$, introduces the highest interferences, and thus the SINR has the lowest values. The rest of the parameters mentioned above have the default values. The differences from one transmit power to another, within this set of results, are about $4 \mathrm{~dB}$ from worst to best case, proving once again that in terms of the influence of the FBS upon the macrocellular layer, the dominating factor is the FBS deployment density, rather than their transmit powers. Considering Algorithm 1, we obtain the best results, with little influence of the FBS transmit power, due to the carrier jumping and remaining on the new carrier, reducing thus the impact of the femtocellullar layer. Algorithm 2 manages to obtain suitable results for the SINR level, within the defined threshold of $-6.5 \mathrm{~dB}$. Using this latter 
International Journal of Wireless \& Mobile Networks (IJWMN) Vol. 4, No. 2, April 2012

algorithm the effect of the transmit power of the FBS is mitigated, thus making it suitable for any case scenario.

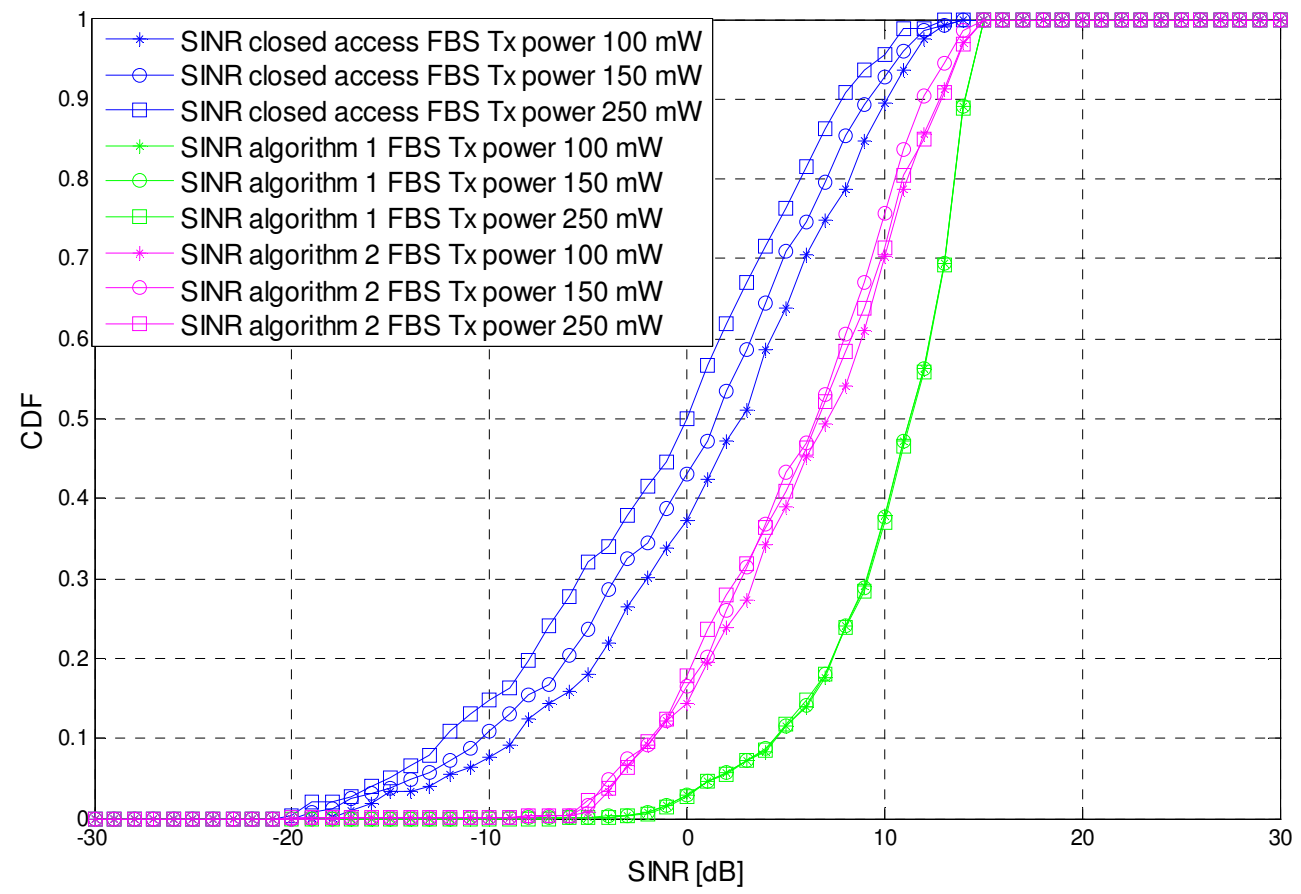

Figure 12. Effect of FBS transmit power.

Because Algorithm 2 manages to fulfil the SINR requirements and offers a better usage of the radio spectrum, in our opinion this is the most suitable option for implementation. Therefore, in the next part of the chapter we will concentrate on this algorithm and investigate how parameters like speed of the user and the come-back time influence its performances. That is why, Figure 13 illustrates the results obtained for the SINR level in terms of the travelling speed of the user. In this case, we consider the default values for all the other parameters.

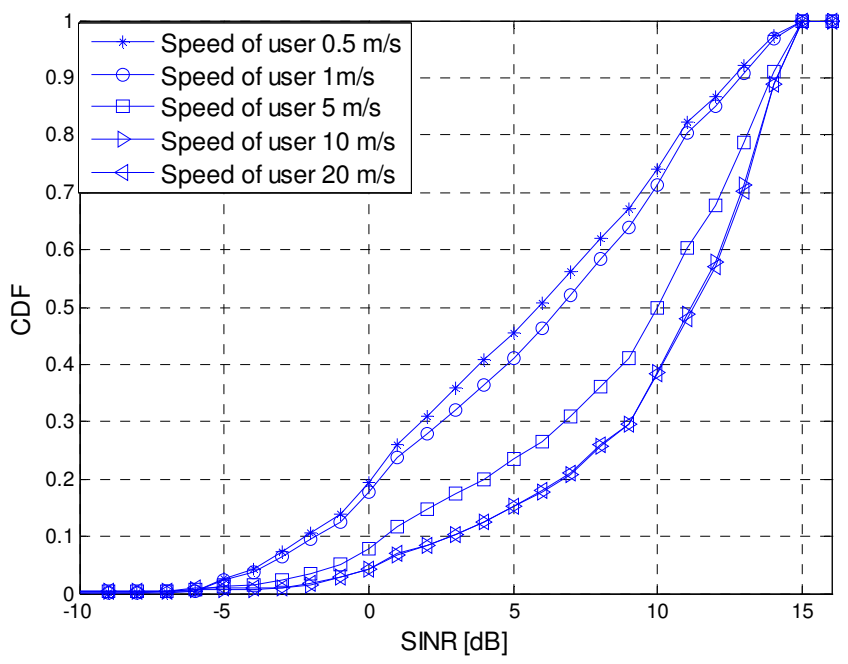

Figure 13. The effect of the user travelling speed. 
International Journal of Wireless \& Mobile Networks (IJWMN) Vol. 4, No. 2, April 2012

One important fact that needs to be mentioned here is that for the simulation cases considered and presented in Figure 13, the SINR conditions are fulfilled, considering the same threshold of $-6.5 \mathrm{~dB}$. The lowest values are obtained for the case when we consider the travelling speed of $0.5 \mathrm{~m} / \mathrm{s}$, simply because the user moves slower and it is subject to interferences for a longer time period. If the travelling speed is increased, in that same amount of time of 5 seconds in which the user is on the second carrier it covers a longer distance, therefore for the same path distance there will be less jumps and less susceptibility to interferences. The best performances are obtained for high speeds, when the user travels its path while being mainly on the second carrier.

Next, we will observe the effect of the come-back time for different values of the travelling speeds of the users. Figure 14 illustrates the effect of the come-back time for four different returning periods: $1 \mathrm{~s}, 2 \mathrm{~s}, 5 \mathrm{~s}$ and respectively $10 \mathrm{~s}$. One may observe that as the returning period after which there is a jump back to the original carrier, increases so does the levels of the obtained SINR.

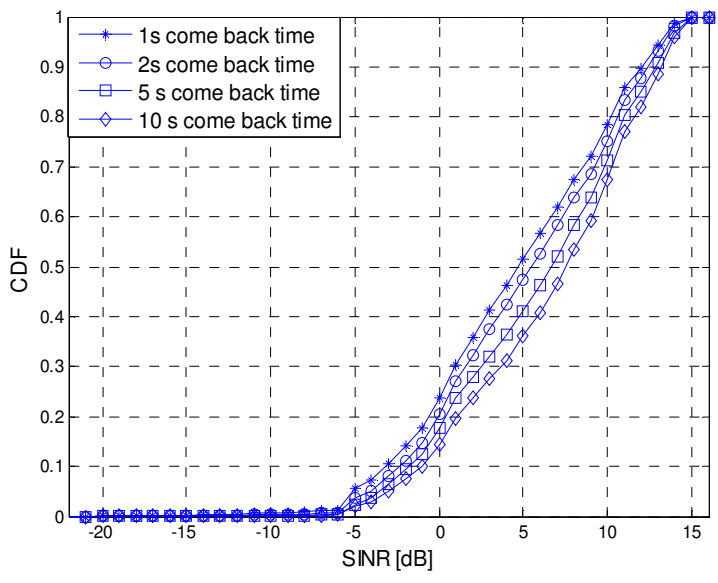

a)

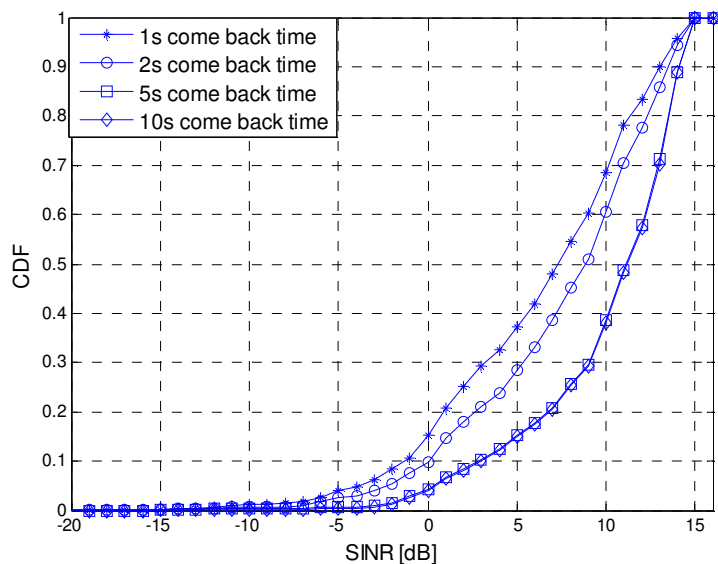

c)

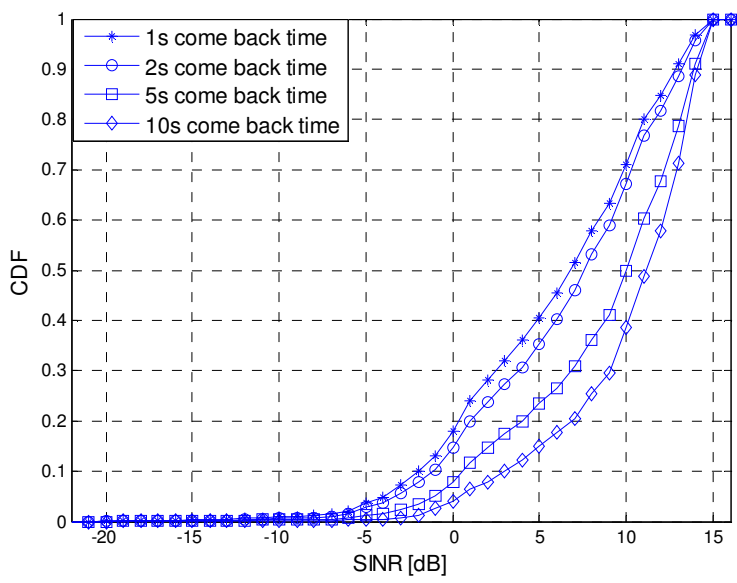

b)

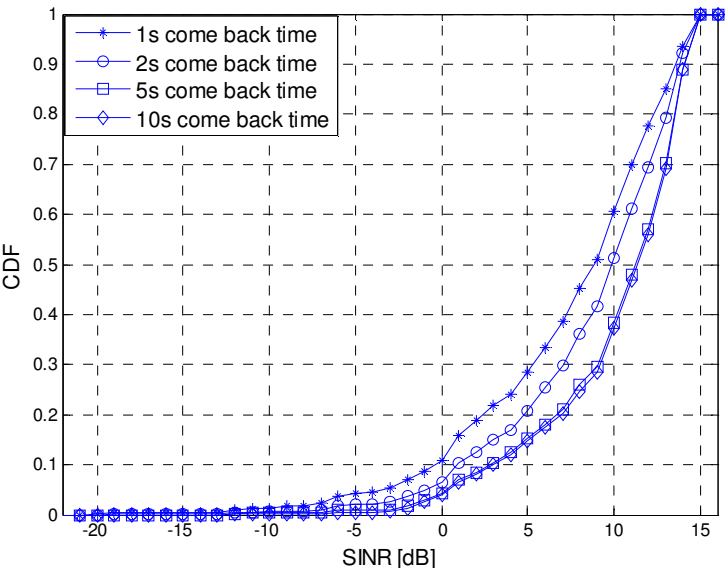

d)

Figure 14. The effect of the come-back time to the original carrier for different travelling speeds: a) $1 \mathrm{~m} / \mathrm{s}$; b) $5 \mathrm{~m} / \mathrm{s}$; c) $10 \mathrm{~m} / \mathrm{s}$; d) $20 \mathrm{~m} / \mathrm{s}$. 
International Journal of Wireless \& Mobile Networks (IJWMN) Vol. 4, No. 2, April 2012

This is due to the fact that if the jump back happens after a shorter period of time, the travelling user will be spending more time on the interference susceptible carrier, thus having lower SINR values. Considering the trend defined by Figure 13, as the speed and come-back time increase, so do the SINR levels. Therefore, the highest performances are obtained for the highest travelling speed of $20 \mathrm{~m} / \mathrm{s}$ and the highest come-back time of 10 seconds.

\section{Conclusions}

The paper managed to investigate in the first part some small cells coverage aspects, in terms of the different parameters that concur to the level of the received signal at the user position. Then, considering a residential environment, we proposed two algorithms that aim at mitigating crosslayer interference, from the femtocell base stations to the macrocellular users that are passing by. We analyzed the performances of the proposed algorithms in terms of the obtained SINR level along the path of the travelling user.

The results show a significant improvement of the SINR level when using either one of the proposed algorithms. Because the first algorithm acts based on a single jump per travelling user, once the channel conditions indicate a jump is suitable and remains on the second carrier, from the spectrum efficiency point of view, this not the best option. In this case, the resulting deployment will be similar to a dedicated carrier approach, which is not desirable.

On the other hand, considering the second algorithm, which imposes a return to the original carrier after a predefined period of time, the obtained SINR level is above the defined threshold value, for more than $99 \%$ of the time, fulfilling thus the QoS requirements imposed by the operator. It offers a higher spectrum efficiency, in this case the second carrier can be viewed as a safety band on which travelling users can be transferred to in order to avoid the high interference. In this way, the operator can assign fewer resources to this second carrier, redirecting most of them to the first carrier.

The proposed solutions offer important enhancements, but still have the disadvantage that they can be applied only for operators that have at least two carriers available for usage. This could be an important option for operators, considering the future benefits they will obtain.

\section{ACKNOWLEDGEMENTS}

This paper was supported by the project "Doctoral studies in engineering sciences for developing the knowledge based society-SIDOC" contract no. POSDRU/88/1.5/S/60078, project co-funded from European Social Fund through Sectorial Operational Program Human Resources 2007-2013.

\section{REFERENCES}

[1] E. Fox, North American ARPU Growth Outpaces the World: A Look at Wireless Forecast Drivers. Yankee Group, March 2006.

[2] H. Claussen, "Co-Channel Operation of Macro- and Femtocells in a Hierarchical Cell Structure", International Journal of Wireless Information Networks, Volume 15, Numbers 3-4, pp 137-147.

[3] H. Claussen, L. T. W. Ho, and L. G. Samuel, "Financial Analysis of a Pico-cellular Home Network Deployment", Procedings of IEEE International Conference on Communications (ICC) 2007, 24-28 June.

[4] Jie Zhang and Guillaume de la Roche, "Femtocells, Technologies and Deployments", Wiley, 2010.

[5] J. Cullen, 'Radioframe presentation,' in Femtocell Europe 2008 , London, UK, June 2008. 
International Journal of Wireless \& Mobile Networks (IJWMN) Vol. 4, No. 2, April 2012

TS 43.318, "Generic Access Network (GAN) Stage 2", Rel-5.

[7] TS 44.318, "Generic Access Network (GAN); Mobile GAN Interface Layer 3 Specification", Rel-5.

[8] TS 25.410, “UTRAN Iu Interface: General Aspects and Principles”, Rel-5.

[9] TS 25.450, “UTRAN IuPC Interface General Aspects and Principles”, Rel-5.

[10] TS 25.419, "UTRAN IuBC Interface: Service Area Broadcast Protocol (SABP)", Rel-5.

[11] Alcatel-Lucent, "The importance of Small Cells in Wireless Networks", Fierce Wireless, April 2011.

[12] 3GPP TR 36.814, "Evolved Universal Terrestrial Radio Access (E-UTRA); Further advancements for E-UTRA physical layer aspects" Version 9, 2010.

[13] C. Androne, T. Palade and E. Puschita, "Open Loop Sensor based System used for Mitigation of Cross-tier Interference in Femtocell Networks ”, TELFOR 2010, Belgrade, Serbia.

[14] M. Z. Chowdhury, Y. M. Jang and Z.J. Haas, "Network Evolution and QoS Provisioning for Integrated Fentocell/Macrocell Networks", International Journal of Wireless \& Mobile Networks, Vol.2, No.3, August 2010.

[15] C. Androne and T. Palade, "Characterization Of Small Cells Networks Deployment Options And Their Impact Upon Macro-Cellular Networks”, Acta Technica Napocensis, Vol.53, No.1, pp. 1520, March 2012.

[16] Mikko Jarvinen, "Femtocell Deployment in 3rd Generation Networks", Master of Science in Technology thesis, Helsinki University of Technology.

[17] ]H. Claussen "Efficient modelling of channel maps with correlated shadow fading in mobile radio systems," Procedings of IEEE Personal, Indoor and Mobile Radio Communication (PIMRC), 2005.

Cristian Androne received his Dipl. Ing. degree from the Technical University of Cluj-Napoca, Romania in 2009. Currently, he is a PhD candidate at the Communications Department of the Technical University of Cluj-Napoca. His current research interests focus on small/macro cells networks deployment integration and optimization.

Tudor Palade received his Dipl. Ing. degree from the Politechnica University of Timisoara, Romania in 1979, and his Ph.D. from the Tehnical University of Cluj-Napoca in 1997. Since 2001 he is a professor at the Communication Department, of the Technical University of Cluj-Napoca. His teaching and research domains include Microwaves Theory, Radio and Cellular Communications, Wireless Systems and Advanced Wireless Architectures. He is a partner in various national and international research projects. 